\title{
Spontaneous uterine rupture of gravid uterus detected on antenatal MRI
}

\author{
Mounisha Kethineni ${ }^{1}$, Venkatesh Manchikanti2* and Sundeep NVK $^{3}$ \\ ${ }^{1}$ Department of Radiology, JJMMC, Davanagere, India \\ ${ }^{2}$ Department of Radiology, Narayana medical college, Nellore, India \\ ${ }^{3}$ Department of Radiodiagnosis, Government General Hospital and Medical college, Ongole, India
}

\begin{abstract}
Spontaneous uterine rupture is a lifethreatening obstetrical emergency encountered infrequently in the emergency obstetric department. Its diagnosis is often missed or delayed, leading to maternal and fetal mortality.
\end{abstract}

\section{Introduction}

Cesarean delivery is a commonly performed major abdominal surgery in women. The surgical incision line in the uterus is generally located in the lower segment of the anterior uterine wall. Although is a safe procedure, cesarean delivery has a variety of complications. The most frequently performed surgical technique is the low transverse Cesarean surgery via a Pfannenstiel or Joel-Cohen skin incision (2-3 $\mathrm{cm}$ above the symphysis pubis), this type of uterine incision is usually recommended because it is related with less postoperative pain and blood loss, and it has the lowest risk for rupture in subsequent pregnancies. Some surgical or obstetric indications may necessitate a classical (i.e., vertical) uterine incision in the body of the uterus; this type of incision is more susceptible to ruptures (3-5.9 per 10,000 pregnancies) [1] and can be associated with more complications for the mother and the baby [2].

\section{Case report}

We present a case of 28-year-old Gravida 2, para 1, live 1 with severe pain in the lower abdomen for 2 days. She had undergone elective lower segment cesarean section (LSCS) 3 years back at 40 weeks of her gestational age. A healthy baby was delivered. She had no medical or antenatal problems. Ultrasound was done at 27 weeks of gestation which revealed a normal fetus with good heart rate. But limited evaluation was possible due to abdominal tenderness and pain on probe compression. However, the pain persisted for a day and hence MRI was advised. MRI was done which revealed an expelled intact amniotic sac with fetus and placenta in the peritoneal cavity (Figure 1) and a focal complete full thickness defect measuring $0.5 \mathrm{~cm}$ noted in the anterior wall of myometrium in the region of previous cesarean scar (Figure 2) and empty endometrial cavity suggestive of uterine rupture. The cervix is empty with normal endocervical canal. The endometrial cavity and endocervical canal are empty.

Emergency laparotomy was performed in view of uterine rupture and persisting severe abdominal pain which revealed fetus with intact amniotic sac in the peritoneal cavity. The placenta was seen underneath the anterior abdominal wall with the fetus lying obliquely

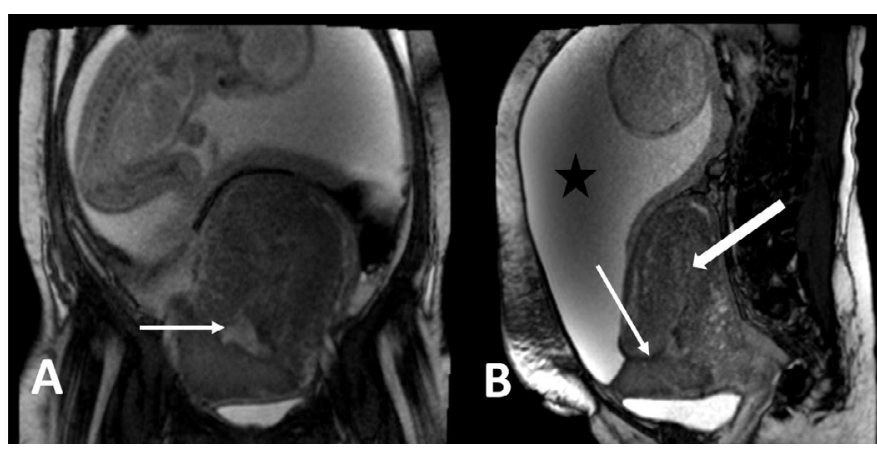

Figure 1(A and B). Coronal and sagittal T2 MRI images demonstrating the amniotic sac with fetus in peritoneal cavity (star). Thin arrows representing the defect in anterior myometrium

close to inferior border of the liver. Fetus was macerated with no cardiac activity.

The extruded baby was delivered by breech extraction along with the placenta. The tear was surgically repaired. She made satisfactory clinical progress and was discharged on the thirteenth postoperative day.

\section{Discussion}

Definitions for uterine rupture are variable, but the most accepted one is that of "those cases of complete separation of the wall of the pregnant uterus with or without expulsion of the fetus." Whenever uterine rupture occurs, there is a significant increase in the maternal and perinatal morbidity and mortality. The chance of fetal survival, especially after rupture and expulsion into the peritoneal cavity, is

*Correspondence to: Venkatesh Manchikanti, Department of Radiology, Narayana medical college, Nellore, India; E-mail: drvenki143@gmail.com

Key words: uterine rupture, MRI, peritoneal cavity, macerated fetus

Received: April 23, 2020; Accepted: May 04, 2020; Published: May 07, 2020 


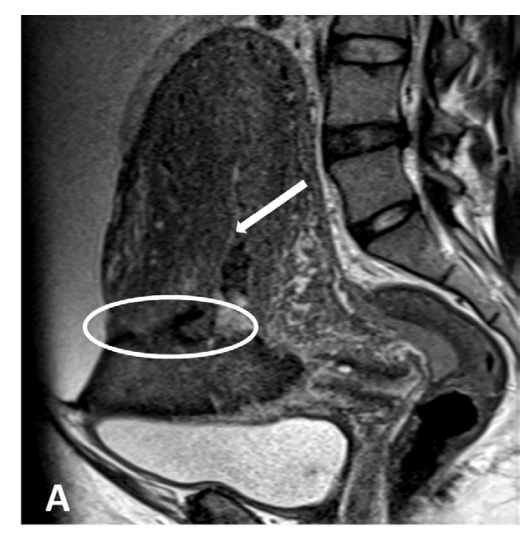

Figure 2. Sagittal T2 MR image showing gravid enlarged uterus full thickness defect in anterior myometrium (oval) and empty endometrial cavity (thick arrow)

dismal, and mortality rates reported in various studies range from 46 to $70 \%$ [3]. There are 3 major categories of uterine rupture: (a) presence of a uterine scar (history of cesarean section or hysterectomy, (b) trauma to an unscarred uterus (breech extraction, forceps, oxytocin, fundal pressure, and shoulder dystocia), and (c) spontaneous rupture of an unscarred uterus [1]. Most cases of uterine rupture in developed countries fall into the first category, which occur in the setting of a uterine scar [1].

When uterine rupture occurs all layers of the uterine wall are separated, including the serosal layer, with full thickness tear and direct communication is seen between the uterus and peritoneal cavity [2]. Rupture of the uterus is a major contributor to maternal and fetal morbidity and mortality, especially in developing countries [3]. Several etiological factors may be responsible for rupture of the uterus. These include previous hysterectomy, cesarean section, trauma, uterine overdistension, uterine anomalies, placenta percreta and choriocarcinoma [3].
Pregnant patients with prior cesarean delivery are also at risk for ectopic pregnancy at the scar, a rare but increasingly reported complication requiring a high degree of suspicion for the diagnosis. Other scar related abnormalities include retained products of conception (RPOC) at the cesarean delivery scar, placenta accreta, malpositioned intrauterine devices (IUDs), and endometriosis. Evaluation of these chronic complications may require US, sonohysterography, magnetic resonance (MR) imaging [4].

\section{Conclusion}

The approach to the pregnant patient with acute abdomen could be very challenging. A wide range of obstetrical and non-obstetrical conditions should be considered, and delay in diagnosis could lead to catastrophic outcome. Uterine rupture should be high on the differential when evaluating abdominal pain in pregnancy especially with prior cesarean delivery [5]. We conclude that MRI is the imaging modality of choice for this population in settings in which MRI is readily available.

In conclusion, clinical suspicion, and systematic approach to acute abdominal pain in pregnancy can prevent misdiagnosis which will help obstetricians in appropriate and immediate surgical intervention.

\section{References}

1. Ponder KL, Won R, Clymer L (2019) Uterine rupture on MRI presenting as nonspecific abdominal pain in a primigravid patient with 28 -week twins resulting in normal neurodevelopmental outcomes at age two. CRIOG 2019: 1-5.

2. Ciolan ER, Negru D, Alecsa-Lupu A-M, Iasi RO (2019) Imagistic evaluation of the uterine scar after cesarean delivery: what the radiologist should know. EPOS 2019: C-2546.

3. Ogbole GI, Ogunseyinde OA, Akinwuntan AL (2008) Intrapartum rupture of the uterus diagnosed by ultrasound. Afr Health Sci 8: 57-59.

4. Rodgers SK, Kirby CL, Smith RJ, Horrow MM (2012) Imaging after cesarean delivery: Acute and chronic complications. RadioGraphics 32: 1693-1712.

5. Moussa HN, Nasab SH, Blackwell SC, Sibai BM (2016) Uterine rupture in pregnancy; Imaging beyond ultrasound. J Neonatal Biol 5: 224.

Copyright: (C2020 Kethineni M. This is an open-access article distributed under the terms of the Creative Commons Attribution License, which permits unrestricted use, distribution, and reproduction in any medium, provided the original author and source are credited. 